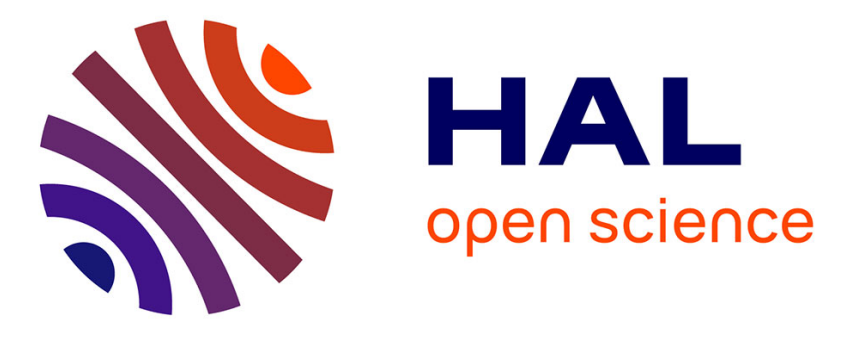

\title{
eHMI positioning for autonomous vehicle/pedestrians interaction
}

\author{
Maureen Troel-madec, Julien Alaimo, Laurence Boissieux, Sandrine \\ Chatagnon, Stan Borkoswki, Anne Spalanzani, Dominique Vaufreydaz
}

\section{- To cite this version:}

Maureen Troel-madec, Julien Alaimo, Laurence Boissieux, Sandrine Chatagnon, Stan Borkoswki, et al.. eHMI positioning for autonomous vehicle/pedestrians interaction. IHM 2019 - 31e conférence francophone sur l'Interaction Homme-Machine, Dec 2019, Grenoble, France. pp.1-8, 10.1145/3366551.3370340 . hal-02388847

\section{HAL Id: hal-02388847 \\ https://hal.science/hal-02388847}

Submitted on 2 Dec 2019

HAL is a multi-disciplinary open access archive for the deposit and dissemination of scientific research documents, whether they are published or not. The documents may come from teaching and research institutions in France or abroad, or from public or private research centers.
L'archive ouverte pluridisciplinaire HAL, est destinée au dépôt et à la diffusion de documents scientifiques de niveau recherche, publiés ou non, émanant des établissements d'enseignement et de recherche français ou étrangers, des laboratoires publics ou privés. 

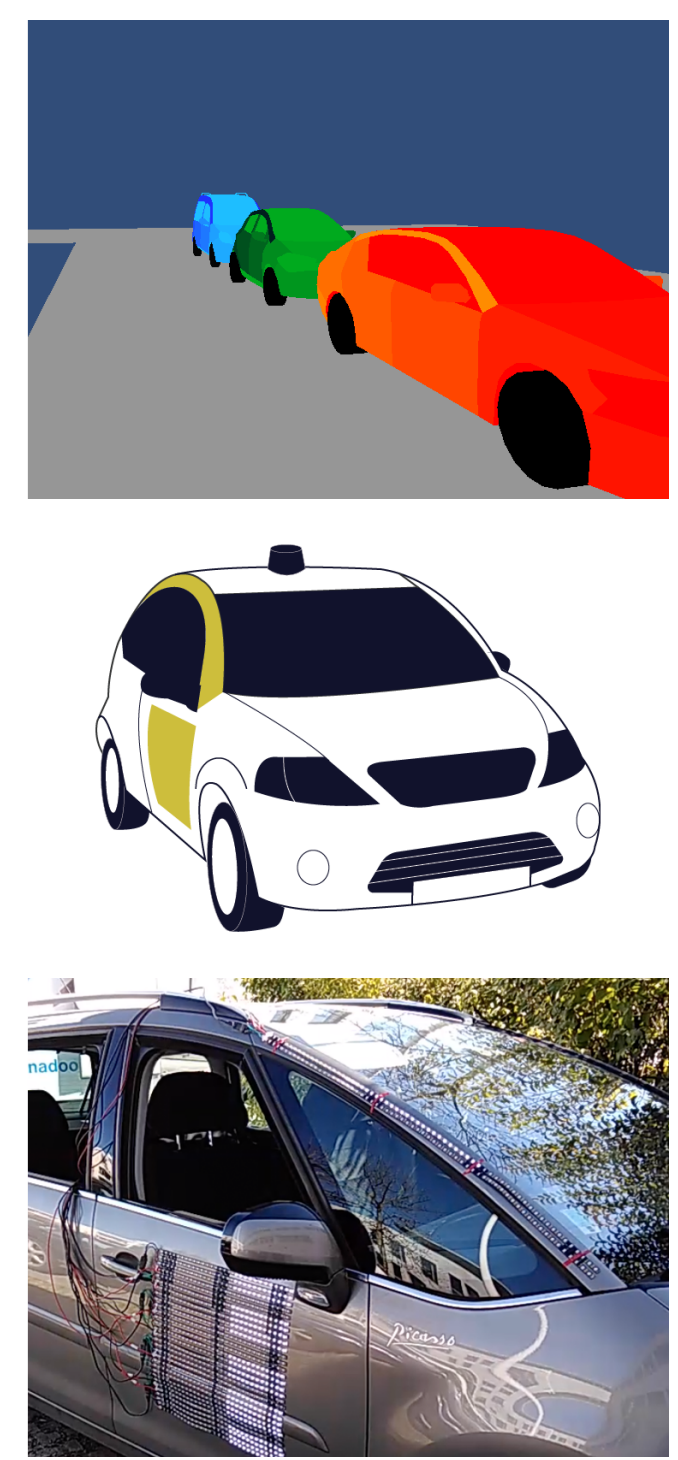

Figure 1: 3D simulation, design and prototype of our external Human-Machine Interface for autonomous car.

\section{eHMI positioning for autonomous vehicle/pedestrians interaction \\ Position du retour visuel pour l'interaction véhicule autonome/piétons}

\section{Maureen Troel-Madec}

Pôle supérieur de design Léonard de Vinci 38090 Villefontaine, France

\section{Laurence Boissieux}

Univ. Grenoble Alpes, Inria, 38000 Grenoble, France

\section{Stan Borkoswki}

Univ. Grenoble Alpes, Inria, 38000 Grenoble, France

\section{Dominique Vaufreydaz}

Univ. Grenoble Alpes, CNRS, Inria, Grenoble INP,

LIG, 38000 Grenoble, France

research@vaufreydaz.org

\section{ABSTRACT}

Progresses on autonomous vehicles suggest a future where they will share urban environments with fragile road users such as pedestrians, cyclists and two wheelers. In this article, we focus on the visual external Human-Machine Interface (eHMI) of autonomous vehicles used while interacting with pedestrians, and more particularly on its placement to increase its relevance and, thus, the safety. We conducted various experiments including 3D simulations to highlight which parts of the (autonomous)

Permission to make digital or hard copies of part or all of this work for personal or classroom use is granted without fee provided that copies are not made or distributed for profit or commercial advantage and that copies bear this notice and the full citation on the first page. Copyrights for third-party components of this work must be honored. For all other uses, contact the owner/author(s)

IHM '19 Adjunct, December 10-13, 2019, Grenoble, France

() 2019 Copyright held by the owner/author(s).

ACM ISBN 978-1-4503-7027-1/19/12.

https://doi.org/10.1145/3366551.3370340

\author{
Julien Alaimo \\ Univ. Grenoble Alpes, Inria, 38000 Grenoble, France
}

\section{Sandrine Chatagnon}

Pôle supérieur de design Léonard de Vinci

\section{Anne Spalanzani}

Univ. Grenoble Alpes, Inria, 38000 Grenoble, France 
vehicles are most prominent in the pedestrian field of view in a scenario where pedestrians cross the road in front of a 3-vehicles row. Our results show that the placement of visual feedback on the front of the vehicle is optimal only if the vehicle is the first one. In other cases, the visibility from the front decreases with the position of the vehicle in the lane. On average on all our simulations, one coarsely can say that the visible parts of the vehicle are $2 / 3$ on the side and $1 / 3$ on the front. In future work, based on this result, we will use the prototype depicted in this article to evaluate the relevance of lateral animations on an autonomous vehicle to communicate its intents to road users.

\section{CCS CONCEPTS}

\section{- Human-centered computing $\rightarrow$ Displays and imagers; • Computer systems organization} $\rightarrow$ External interfaces for robotics.

\section{KEYWORDS}

interaction technique, autonomous vehicle, external Human-Machine Interface (eHMI)

\section{INTRODUCTION}

To be autonomous, aka self-driving, a vehicle needed to locate itself, to perceive its environment, to decide on the best trajectory and to operate its actuators to follow that trajectory in a process known as control loop. Recently, an interest emerged in using more elaborated information and producing finer and even social behaviours [13], particularly for autonomous vehicle management in urban city centers. In this context, an autonomous car handles way more complex environments and actions. More than a control loop, today an autonomous vehicle is enabled with an interaction loop. Its numerous interaction partners include smart-cities and smart-roads that will exchange data with the vehicle. This information is local data captured by so-called loT systems $[12,18]$ for traffic, pollution, parking management... The other (autonomous) vehicles will also share their gathered data $[10,20]$ (positions, perception of the environment, etc.) and cooperate to decide on a collective behaviour combining safety and efficiency. Finally, the last partners in interaction are the other road users, namely drivers, cyclists and pedestrians.

One way to increase safety for VRU is to enable autonomous cars with an External Human-Machine Interface (eHMI) to complete the interaction loop by providing valuable feedback to pedestrians about perception, decisions and intents of the vehicle [14, 19, 21, 23]. This feedback could broaden the reading level of vehicle behaviours in interaction situations. In this article, we will focus on this eHMI, and more specifically on its location to maximize its visibility by pedestrians, hence enhancing the safety and the interaction performance in urban center scenarios. 

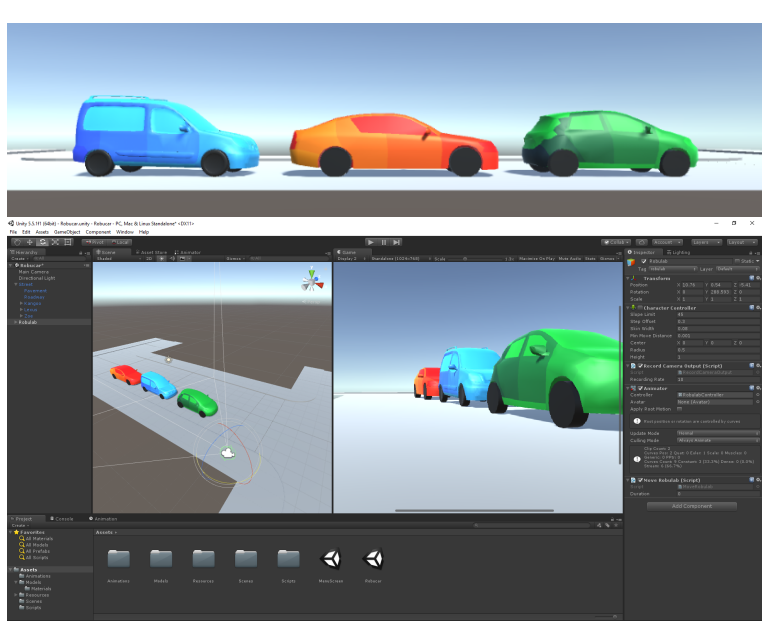

Figure 2: 3D simulation framework. At top, a side view of the 3 cars. At bottom, the scene setup in Unity3D including the visualization and images generation parts. The simulator handles pedestrian simulated trajectories and point of views. Usage of a flat color shader to generate the images allows to easily segment and identify the visible parts of each car.
The article is organized as follow. We first present related work about eHMI for autonomous vehicles. In the next section, experiments are depicted and analysed. The developed prototype and conclusion are exposed in the last sections.

\section{RELATED WORK}

Industrialists have been working on eHMI prototypes allowing interactions between these vehicles and the pedestrians. Different systems have been designed by car manufacturers and researchers using mainly two technologies for eHMI implementation [14, 19, 21, 23]. The first one is the projection of surrounding information on the road to let pedestrians know what their options are (cross the road, wait, signal their intention, etc.) according to the vehicle's perception of the environment [2, 4-6]. It can also help to indicate more clearly the decisions taken by the vehicle $[11,17]$ (accelerate, brake, avoid by the right...). The second approach concerns the integration of LED displays positioned on the vehicle, presenting the information formerly mentioned, i.e. pictograms or texts elucidating the intention of the vehicle. These displays are frequently placed on the bumpers $[5,7,25]$ in the front and/or rear of the car, on the hood [15] or at the bottom or top of the windshield [1, 21]. Few concepts propose visual feedback on the sides of the car $[3,8]$. The positioning of these devices on the vehicle is almost never debated. The design is generally depicted without justification, sometimes it looks like it was inspired by science fiction or movies [17], sometimes the design has an anthropomorphic purpose $[5,9,16,22]$, etc. For instance, in the case of the smiling car [5], the LED display is on the front bumper and offers variations of a smile. This smile is completed with the vehicle's headlights to suggest a "face" on the vehicle. As we said in the introduction, it appears that this positioning, without considering the anthropomorphic aspect in this specific case, raises questions about its ability to transmit necessary information in various situations.

\section{EXPERIMENT}

Experiments conducted either using Wizard of Oz [24], videos [14] or augmented reality [17] paradigms present the autonomous vehicle alone or first in the row. As a consequence, there is no problem to see its front bumper and hood (and respectively for the rear parts). However, in urban scenarios involving autonomous vehicles, this may not necessarily be the case as illustrated at top on Figure 1. This simulation shows 3 vehicles in a single row and the point-of-view of a pedestrian arriving at the side of the road, wishing to cross. If the autonomous vehicle is second or third, the front parts are hidden due to perspective effect and other cars. The frontal display becomes useless in this case.

We carried out an experiment to study the optimal position of the display, i.e. the position maximizing its visibility in all conditions to ensure maximum feedback for pedestrians and to increase safety. First, using a $360^{\circ}$ camera, we simulated the point of view of a pedestrian crossing in front a several cars. After analysing the videos, we concluded that that positioning the eHMI optimally is not 
an easy task. We decided to deeper investigate by conducting 3D simulations to tangibly measure which parts were most visible to pedestrians. The 3D virtual scene consists in a double lane street bordered with sidewalks on each side. Three cars are aligned on the right lane of the street. We choose to keep the scene as simple as possible, without any street furniture nor buildings, to optimize the computation of visible parts. We feature cars of three main categories: a city car (green), a sedan (red) and a van (blue). Each one has a main color declined in variations according to each part of the car (see figures 1 and 2). The 3D simulations were done by varying the vehicles placement in a row of 3 cars, the types of pedestrians (children/teenagers/adults, i.e. the height of their eyes from $1 \mathrm{~m}$ to $1.8 \mathrm{~m}$ with a step of $0.2 \mathrm{~m}$ ) and the direction of their gaze (straight ahead when crossing the road or looking at the autonomous vehicle). To obtain a substantial dataset, we ran a total of 332 simulations varying these parameters and making the pedestrian cross the street from left to right and vice versa.

After the simulation process, we computed for each vehicle (the first, second and third in the row) the percentage of each visible part separately, and then their global percentages for all 3 cars. This percentage corresponds to the visible surface (i.e. number of pixels) of part $p$ of car $c$ out of the total surface of the car. It is computed using the following formula:

Table 1: Average percentage of visible parts of the vehicles. Results are given averaging all simulations for the front car, the middle car, the rear car and the global score for all cars.

\begin{tabular}{lcccc}
\cline { 2 - 5 } & Front car & Middle car & Rear car & All cars \\
\hline Front & $54,04 \%$ & $26,37 \%$ & $2,05 \%$ & $30,57 \%$ \\
\hline Side & $45,21 \%$ & $65,16 \%$ & $85,25 \%$ & $63,15 \%$ \\
\hline Rear & $0,75 \%$ & $8,46 \%$ & $12,70 \%$ & $6,29 \%$ \\
\hline
\end{tabular}

Visible_percentage $(p, c)=$ Number_of_visible_pixels $(p, c) /$ Total_number_of_visible_pixels $(c)$

Table 1 summarizes the gathered results grouping the car body parts: the front parts (bumper, bonnet, windscreen), the side parts (door and side rail above it) and the rear parts (bumper and rear trunk). If we consider the results in this table, we first see a trivial result: for the head-of-line vehicle, more than half of the visible surface (55\%) in the pedestrian field of view is concentrated on the front part of the vehicle. Note that $45 \%$ of its visible surface also concerns the side of the vehicle, while the rear part is poorly visible. For the second vehicle on the line, the visibility rate of the front end decreases to nearly $26 \%$. This is mainly due to the fact that it is hidden by the first vehicle. Side visibility increases to $65 \%$, which is becoming the majority. The last vehicle follows the same logic. Its visible frontal surface is even smaller $(2 \%)$ because in addition to the vehicle's masking in front of it, there is an increase of the perspective effect. Looking at the last line of Table 1, we see that the rear part of the vehicles is poorly represented. Nonetheless, it can still represent up to $12 \%$ of the visible surface of a vehicle in a row. If we summarize eluding the rear part of the vehicle, when a pedestrian crosses in front of 3 cars in a row, the first vehicle is visible half face and half side, the second $1 / 3$ face and $2 / 3$ side. For the third one, the lateral part becomes prominent with a visibility rate of $85 \%$. 


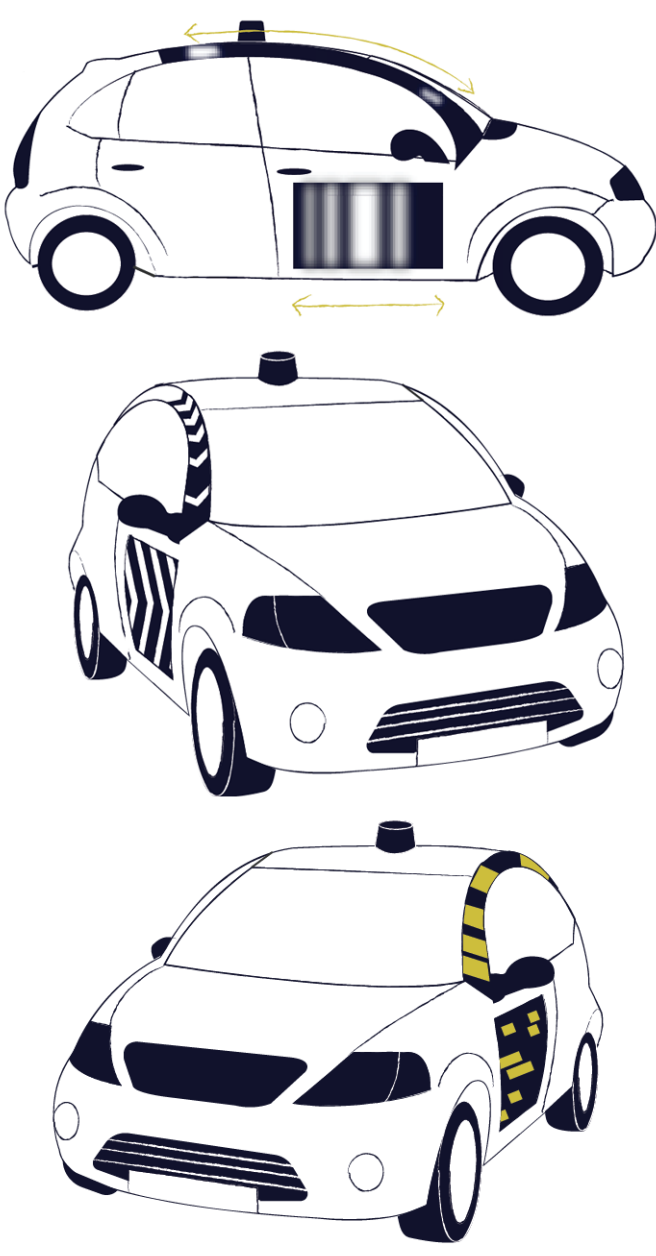

Figure 3: Thoughts on using lateral surfaces to express car speed through animated coloured patterns.

\section{DESIGN AND EXPERIMENTAL PROTOTYPE}

\section{Design hypotheses}

Following the results of visibility presented in the previous section, we considered several typical scenarios between pedestrians and autonomous vehicles where only the lateral parts of the vehicles would be used for communication. Once again, we do not dispute the relevance of a front display for the first car. But statistically for the general case, the lateral parts of the vehicle are the most visible parts and we would like to focus on them in the first stage. Using role-plays and working groups with road users, we made several hypotheses concerning the visual rendering of autonomous vehicles during interaction with pedestrians. As in a patent registered by Google in 2015 [26], we were first interested in the use of complex visuals on side doors. This solution has the advantage of being very effective at short range. It is possible to use complex pictograms or texts indicating clear actions such as "you can cross the street in front of me". (see figure 5). However, this solution has a major drawback when the vehicle is distant: reading these signals becomes difficult for pedestrians. In a study using still images [14], it is shown that the colour and textual content are also significant.

We conducted a survey on the communication possibilities using animations. They could replace the pictograms addressing the problems of perspective and distance while adding readability. An animal communication model caught our interest: the octopus model. These animals use variations in patterns, colours and brightness to communicate (love parade, territory defence, etc.). We integrated this communication model to imagine new forms of blinking or variations in colour and/or patterns that could be interpreted by humans from far away. After exchanges with road users, we decided to extend our display to the upper side rail along the windscreen and doors 3 , which is visible from a long distance (15\% of the visible part of the 3rd vehicle in our simulations). At this stage of our thinking, 2 steps remained to be carried out to finalize our study. Build a prototype and evaluate our hypotheses in real conditions with users. The prototype has been developed and is the subject of the following section. Using it, we defined several types of coloured light patterns that, we hope, will allow us to communicate vehicle decisions to other road users in the same way as octopuses (Figure 3 depicts some of these ideas).

\section{Experimental device}

Knowing that the current design of our prototype has not been experimentally validated yet, we may reconsider some of our design hypotheses, including form and location of the display. We chose to develop a hardware allowing us to create free shapes for our external display. Using flexible LED ribbons as underlying pixel technology allows us to cover non-flat areas. The hardware solution developed is based on the FadeCandy open source electronic controllers (https://github.com/scanlime/fadecandy) that enables a large number of LED ribbons to be connected. These are controlled via USB by a 
computer, a Raspberry-pi being sufficient for this purpose. In our prototype, it is possible to use more than 21K LEDs with a single device. By multiplying the devices, we can further increase this ceiling. The real technical limit to the use of these prototypes is the electrical power that must be delivered if all LEDs are being illuminated simultaneously in pure white, the most energy consuming colour. It is therefore necessary to integrate this constraint into the design of our animations in order not to overload the electrical circuit. The device works with $12 \mathrm{~V} / 5 \mathrm{~V}$ converters that allow easy inclusion in our vehicle. Several types of ribbons are available varying in length, LED density and waterproof rating. To fix them on the car, we designed 3D-printable fixations including strong neodymium magnets or fastening strap. The control software has been developed in Python. It can play on demand prerecorded animations at a frequency up to $200 \mathrm{hz}$ maximum, depending on the number of LED. It is also able to play animations computed on-the-fly and sent by a remote computer, which allows the animations to be adapted online to the perceptions/decisions of the vehicle. These animations can be played on LED ribbon assemblies of any shape. Ribbons can be cut or soldered to reduce or increase their length. As an example, in the current prototype (see bottom of Figure 1), the display surface consists of a $30 \times 30$ pixel area $(45 \times 45 \mathrm{cms})$ on the door and a $2 \times 70$ pixel area $(3 \times 105 \mathrm{cms})$ on the side-rail.

The control software, the fixing parts 3D models as well as the fabrication plans of our device will be made available online to the community in open source. This will allow researchers to reproduce our experiments but also to develop all types of LED displays for any types of research.

\section{take away}

In our scenario of pedestrians crossing in front of a 3-car row, averaging on all 3D simulations, the side parts represent $2 / 3$ of the visible surfaces, $1 / 3$ for the front parts of the vehicle.

This confirms the interest of having an eHMI in the front part of the vehicle (mostly for the front car) but clearly indicates that in urban scenarios, it is indispensable to complete it with lateral displays.

\section{CONCLUSION}

In this article, we addressed the problem of positioning an external human-machine interface for interactions between an autonomous vehicle and the surrounding pedestrians. We demonstrated through 3D simulations that the location on the front of the vehicle may not be ideal in urban scenarios. Indeed, our results showed that in the presence of multiple in-line vehicles, the most visible part is not the front but the side of the car. When a pedestrian crosses the street in front of a row of vehicles, he perceives in his field-of-view the front and side part roughly at $50 \% / 50 \%$ for the first vehicle, at $33 \% / 66 \%$ and $15 \% / 85 \%$ respectively for the second and third car. This effect increases as the vehicle moves back into the queue and therefore seems to indicate that for more distant vehicles, it would be the same. This conclusion does not affect the relevance of a front display on the car but clearly indicates that it is not sufficient in urban environments.

To validate our findings and the actual positioning of visual feedback on the autonomous vehicle, we designed a prototype and its associated animations using only the lateral part of the car. This design has led to the development of a flexible and scalable hardware device and a control software that will be made available to the community in open source. This device was technically validated by a few users during an experimental protocol using videos of a static vehicle. We must now go ahead on future experiments conducted with a prototype embedded on a real moving car in ecological conditions 
by evaluating animations against several criteria: visibility in real conditions and appropriateness to express the vehicle's intents for the safety aspect, not forgetting the power consumption and the potential generated visual pollution for the environmental considerations.

\section{ACKNOWLEDGMENTS}

This research has been funded by the French ANR project HIANIC (ANR-17-CE22-0010), and was made possible by the use of equipment provided by ANR Equipment for Excellence Amiqual4Home (ANR-11-EQPX-0002).

\section{REFERENCES}

[1] 2014. AutonoMI Autonomous Mobility Interface. https://vimeo.com/99160686. (2014). [Online; accessed 19-August-2015].

[2] 2015. AutoMotoTV: Mercedes-Benz F 015 Luxury in Motion - projection on the road. https://www.youtube.com/watch?v= FnU6gZ6LIAo and https://www.youtube.com/watch?v=fvtlobbMENo. (2015). [Online; accessed 19-August-2015].

[3] 2015. IDS Concepte. https://www.nissan.co.uk/experience-nissan/concept-cars/ids-concept.html. (2015). [Online; accessed 19-August-2015].

[4] 2015. Mitsubishi Electric: road-illuminating directional indicators. https://www.mitsubishielectric.com/news/2015/1023. html. (2015). [Online; accessed 19-August-2015].

[5] 2016. Semcon: Who sees you when the car drives itself? https://semcon.com/smilingcar/. (2016). [Online; accessed 19-August-2015].

[6] 2017. Rinspeed AG. https://www.rinspeed.eu/en/Oasis_21_concept-car.html. (2017). [Online; accessed 19-August-2015].

7] 2018. Concept-i. https://newsroom.toyota.eu/2018-toyota-concept-i. (2018). [Online; accessed 19-August-2015]

[8] 2018. Drive-ai concept car. https://www.drive.ai/. (2018). [Online; accessed 19-August-2015].

[9] 2018. Virtual eyes haveit. https://www.jaguarlandrover.com/2018/virtual-eyes-have-it. (2018). [Online; accessed 19-August2015].

[10] Mohammad Y Abualhoul, Pierre Merdrignac, Oyunchimeg Shagdar, and Fawzi Nashashibi. 2016. Study and evaluation of laser-based perception and light communication for a platoon of autonomous vehicles. In 2016 IEEE 19th International Conference on Intelligent Transportation Systems (ITSC). IEEE, 1798-1804.

[11] Claudia Ackermann, Matthias Beggiato, Luka-Franziska Bluhm, Alexandra Löw, and Josef F Krems. 2019. Deceleration parameters and their applicability as informal communication signal between pedestrians and automated vehicles. Transportation research part F: traffic psychology and behaviour 62 (2019), 757-768.

[12] Furqan Alam, Rashid Mehmood, lyad Katib, Nasser N Albogami, and Aiiad Albeshri. 2017. Data fusion and loT for smart ubiquitous environments: a survey. IEEE Access 5 (2017), 9533-9554.

[13] Self citation. 2019. . In . IEEE, 1-7.

14] Pavlo Bazilinskyy, Dimitra Dodou, and JCF De Winter. 2019. Survey on eHMI concepts: The effect of text, color, and perspective. Manuscript submitted for publication (2019).

[15] Ola Benderius, Christian Berger, and Victor Malmsten Lundgren. 2017. The best rated human-machine interface design for autonomous vehicles in the 2016 grand cooperative driving challenge. IEEE Transactions on intelligent transportation systems 19, 4 (2017), 1302-1307.

[16] Chia-Ming Chang, Koki Toda, Daisuke Sakamoto, and Takeo Igarashi. 2017. Eyes on a Car: an Interface Design fo Communication between an Autonomous Car and a Pedestrian. In Proceedings of the 9th International Conference on Automotive User Interfaces and Interactive Vehicular Applications. ACM, 65-73. 
[17] Koen de Clercq, Andre Dietrich, Juan Pablo Núñez Velasco, Joost de Winter, and Riender Happee. 2019. External human-machine interfaces on automated vehicles: effects on pedestrian crossing decisions. Human factors (2019), 0018720819836343.

[18] S Alireza Fayazi and Ardalan Vahidi. 2017. Vehicle-in-the-loop (VIL) verification of a smart city intersection control scheme for autonomous vehicles. In 2017 IEEE Conference on Control Technology and Applications (CCTA). IEEE, 1575-1580.

[19] Ann-Christin Hensch, Isabel Neumann, Matthias Beggiato, Josephine Halama, and Josef F. Krems. 2019. How Should Automated Vehicles Communicate? - Effects of a Light-Based Communication Approach in a Wizard-of-Oz Study. In Advances in Human Factors of Transportation, Neville Stanton (Ed.). Springer International Publishing, Cham, 79-91.

[20] Sampo Kuutti, Saber Fallah, Konstantinos Katsaros, Mehrdad Dianati, Francis Mccullough, and Alexandros Mouzakitis. 2018. A survey of the state-of-the-art localization techniques and their potentials for autonomous vehicle applications. IEEE Internet of Things Journal 5, 2 (2018), 829-846.

[21] Tobias Lagstrom and Victor Malmsten Lundgren. 2015. AVIP-Autonomous vehicles interaction with pedestrians. Master of Science Thesis, Chalmers University of Technology (2015).

[22] Nicholas Pennycooke. 2012. AEVITA: designing biomimetic vehicle-to-pedestrian communication protocols for autonomously operating \& parking on-road electric vehicles. Ph.D. Dissertation. Massachusetts Institute of Technology.

[23] Amir Rasouli and John K. Tsotsos. 2018. Autonomous Vehicles that Interact with Pedestrians: A Survey of Theory and Practice. CoRR abs/1805.11773 (2018). arXiv:1805.11773 http://arxiv.org/abs/1805.11773

[24] Dirk Rothenbücher, Jamy Li, David Sirkin, Brian Mok, and Wendy Ju. 2016. Ghost driver: A field study investigating the interaction between pedestrians and driverless vehicles. In 2016 25th IEEE international symposium on robot and human interactive communication (RO-MAN). IEEE, 795-802.

[25] Matthew Sweeney, Thomas Pilarski, William Payne Ross, and Chenggang Liu. 2018. Light output system for a self-driving vehicle. (Dec. 25 2018). US Patent App. 15/803,184.

[26] Christopher Paul Urmson, Ian James Mahon, Dmitri A Dolgov, and Jiajun Zhu. 2015. Pedestrian notifications. (Feb. 10 2015). US Patent $8,954,252$ 